\title{
Infectious transmission from Savary dilators is uncommon: retrospective review from a large tertiary academic center
}

\author{
Zoe N. Memel ${ }^{1,2}$, Duncan J. Flynn ${ }^{1,2}$, Yasmin Hernandez-Barco ${ }^{1,3}$, Kavel Harish Visrodia ${ }^{1,3}$, Brenna Casey ${ }^{1,3}$, Kumar Krishnan ${ }^{1,3}$ \\ ${ }^{1}$ Harvard Medical School, Boston; ${ }^{2}$ Department of Medicine, Massachusetts General Hospital, Boston; ${ }^{3}$ Division of Gastroenterology, Massachusetts General \\ Hospital, Boston, MA, USA
}

In recent years, there have been numerous multi-drug resistant outbreaks attributed to endoscopes, with studies documenting residual bioburden on endoscopes, suggesting inadequate disinfection as the cause of these outbreaks. ${ }^{1,2}$ Wire-guided bougie dilation (WBD) is a common treatment strategy for esophageal strictures. Similar to gastrointestinal endoscopes, dilators have long, narrow lumens, and high-level disinfection (HLD) is used to reprocess these devices. However, despite the breadth of data on reprocessed endoscopes, there are no data on the prevalence of infectious transmission between reprocessed WBD dilators. Given the exposure of these instruments to the microbial bioburden in the oral cavity and esophagus, we sought to determine whether WBD dilation poses the same potential risk.

Based on the few studies performed on the reprocessing of flexible endoscopes, the increased level of pathogen transmission has been linked to inadequate cleaning, incorrect selection of high-level disinfecting agents, and/or improper drying and other breeches in reprocessing. ${ }^{3-5}$ Given the paucity of data evaluating the infectious transmission rate with reusable WBD dilators, the objectives of this study were (1) to determine the rate of infectious complications after use of WBD dilators during endoscopic procedures, and (2) to evaluate the etiology of re-

\footnotetext{
Received: January 18, 2021 Revised: March 16, 2021

Accepted: March 21, 2021

Correspondence: Zoe N. Memel

Massachusetts General Hospital, Gastroenterology Division, 55 Fruit Street, Boston, MA 02114, USA

E-mail: zmemel@mgh.harvard.edu
}

(a) This is an Open Access article distributed under the terms of the Creative Commons Attribution Non-Commercial License (http://creativecommons.org/licenses/by-nc/4.0/) which permits unrestricted non-commercial use, distribution, and reproduction in any medium, provided the original work is properly cited. admission within 90 days following endoscopy with the use of WBD dilators.

Billing data from the Department of Gastroenterology, Massachusetts General Hospital were utilized to retrospectively identify patients who underwent endoscopy with WBD to treat esophageal strictures from 2000 to 2019. An infectious complication was defined as any infectious process that developed in a patient within 90 days after their endoscopic procedure. $\mathrm{Pa}$ tients who had esophageal stents placed during the same time period were excluded. Cases were examined for primary demographic data, immediate complication rates, and readmission within 90 days of the procedure with a review of all admission notes, emergency department visits, endoscopy reports, relevant progress notes, microbiology results, and discharge summaries. Data extracted included readmission etiology and possible confounding factors, including the recent use of immunosuppressive medications, antibiotics, chemotherapy, radiation, and any active infections at the time of the dilation. Reasons for readmission were grouped into infectious and non-infectious etiologies. The results were categorized into subgroups, and the percentage of each etiology for readmission was calculated. The bougie dilators evaluated in this study were manufactured by two manufacturers. Savary-Gilliard dilators were made by Cook Medical Device Company (Billerica, MA, USA), and American dilators were made by ConMed (Westborough, MA, USA). Our institutional protocol for WBD reprocessing utilizes ortho-phthalaldehyde for HLD; further information on the specific reprocessing protocol is detailed in Supplementary Material 1. This study was conducted in full accordance with all applicable Massachusetts General Hospital Policies and Procedures. 
In total, 528 patients underwent WBD and met the inclusion criteria. Forty-four $(8.3 \%)$ of these patients were readmitted within 90 days of the procedure (Table 1), of which eight had infections defined clinically or radiographically (18.2\%). The most common infection was respiratory etiology $(n=7,15.9 \%)$, followed by gastrointestinal ( $n=1,2.3 \%)$. Gastrointestinal (GI) infection was secondary to Clostridium difficile. The remaining 35 cases of readmission within the first 90 days (79.5\%) were due to cardiac etiologies, non-infectious GI-related symptoms (nausea, vomiting, dysphagia), metabolic abnormalities (such as hyponatremia), and miscellaneous etiologies (including falls, alternate non-gastrointestinal procedures, etc.). The mean duration between procedure and readmission was 34.5 days.

In reviewing the specific dilators used in each case, none of the same-sized dilators were used within a three-month period of another case with an infectious complication. Our Depart- ment of Gastroenterology has two complete sets of bougie dilators that range from 18 to 60 French within the endoscopy unit. We estimate that we average 1 dilation every 14 days and estimate that the same dilator is used every 30 days as a conservative approximation. In total, out of the patients readmitted within 90 days, four patients had undergone radiation within three months of the procedure, 11 patients had undergone both chemotherapy and radiation within three months of their procedure (25.0\% of readmissions), and two patients had been taking an immunosuppressant agent, specifically mycophenolate mofetil or etanercept, prior to the procedure $(4.5 \%$ of readmissions). Of the patients who underwent any form of chemotherapy, radiation, or immunosuppressive regimen, only two developed infections, both of which were respiratory in origin. Additionally, 7 patients (15.9\%) had taken antibiotics within 3 months prior to the procedure. Of note, the patient diagnosed

Table 1. Characteristics and outcomes of patients who had readmission to a hospital within 90 days after esophageal dilation using wire-guided bougie dilation

\begin{tabular}{|c|c|}
\hline Characteristic & Value \\
\hline Total participants with readmission after EGD within 90 days & $44(8.3 \%$ of total participants, $n$ total $=528)$ \\
\hline Female & $27(61.4)$ \\
\hline Male & $17(38.6)$ \\
\hline Mean age (yr) & 68.5 \\
\hline Average days to readmission (day) & 34.5 \\
\hline \multicolumn{2}{|l|}{ Indication for esophagogastroduodenoscopy } \\
\hline Benign esophageal stricture & $33(75.0)$ \\
\hline Malignant esophageal stricture & $7(15.9)$ \\
\hline Cervical web/Schatzki ring & $4(9.1)$ \\
\hline \multicolumn{2}{|l|}{ Factors affecting patients' risk for post-dilation complication } \\
\hline Antibiotics within 3 months prior to procedure & $7(15.9)$ \\
\hline Active infection at time of procedure & $3(6.8)$ \\
\hline Immunosuppressant agent use at time of procedure & $2(4.5)$ \\
\hline Undergoing radiation at time of procedure & $4(9.1)$ \\
\hline Undergoing chemotherapy at time of procedure & $0(0)$ \\
\hline Undergoing chemotherapy \& radiation at time of procedure & $11(25.0)$ \\
\hline \multicolumn{2}{|l|}{ Readmission for infectious diagnoses } \\
\hline Total infectious readmissions & $8(18.2)$ \\
\hline Respiratory infection & $7(15.9)$ \\
\hline Gastrointestinal infection & $1(2.3)$ \\
\hline \multicolumn{2}{|l|}{ Readmissions for non-infectious diagnoses } \\
\hline Total non-infectious readmission & $35(79.5)$ \\
\hline Cardiac diagnosis & $7(15.9)$ \\
\hline Gastrointestinal diagnosis & $15(34.1)$ \\
\hline Metabolic abnormality & $5(11.4)$ \\
\hline Miscellaneous diagnosis & $12(27.3)$ \\
\hline
\end{tabular}

Values are presented as number (\%). 
with $C$. difficile was not taking antibiotics prior to the procedure. Three patients had active infections at the time of their WBD dilations: one had a urinary tract infection, while two had chronic obstructive pulmonary disease exacerbations presumed to be due to respiratory tract infections. Patients with an infectious complication were readmitted on average 35.7 days after their procedure. To our knowledge, this is the first retrospective case series to assess the incidence of infectious complications and readmission after undergoing WBD. The findings of this study demonstrate that the 90-day readmission rate for infectious complications after WBD was low at approximately $8 \%$. A small but finite risk of pulmonary and gastrointestinal infections was observed; however, based on analysis of the timeline of dilation among patients with consecutive infections, the likelihood of cross-contamination appears low. In previous endoscopy-associated transmission cases, transmission typically occurred within several weeks of contamination, ${ }^{1,2}$ and despite our 90-day window, the majority of cases occurred in the first 30 days, consistent with prior studies. The Spaulding classification system is utilized by the Centers for Disease Control and Prevention ${ }^{6}$ and the US Food and Drug Administration ${ }^{7}$ to categorize medical devices according to their risk of infection. A semi-critical device is one that contacts intact mucous membranes, such as endoscopes and WBDs. Devices within this class should receive high-level disinfection, defined as the destruction of all vegetative microorganisms, mycobacteria, small nonlipid viruses, medium lipid viruses, fungal spores, and some, but not all, bacterial spores. ${ }^{8,9}$ Although there remains a lack of consensus on the clinical value of routine microbiological monitoring of flexible endoscopes, there has been little discussion on the utility of monitoring the microbiological bioburden level on WBD. Current reprocessing guidance by the American Society of Gastrointestinal Endoscopy and the Association for the Advancement of Medical Instruments do not specifically address endoscopic dilators. ${ }^{9}$ However, the latter is anticipated to release a new informational, technical report, TIR99, specifically addressing the reprocessing of dilators, ultrasound, and manometry probes. ${ }^{10}$ The limitations of this study include its retrospective study design, lack of residual bioburden culture data, and inability to provide further information on dilator specifics (such as purchase date and number of uses). Given our inability to perform residual bioburden cultures, there is insufficient evidence to directly evaluate WBD transmission of infection.

In conclusion, the risk of infection-associated readmission within 90 days after WBD was low. Moving forward, well-designed studies measuring the incidence of pathogen-associated transmission during endoscopic procedures using WBD are needed to better understand the rate of infectious transmission and the efficacy of HLD in minimizing the residual bioburden on these instruments.

\section{Supplementary Material}

Supplementary Material 1. Bouige dilator and wire (non-disposable) reprocessing guide: Massachusetts General Hospital White 3 Endoscopy \& Charles River gastrointestinal outpatient reprocessing rooms guidelines.

Supplementary material related to this article can be found online at https://doi.org/10.5946/ce.2021.050.

\section{Conflicts of Interest}

Yasmin Hernandez-Barco is Public analysis Inc in Behalf of Takeda, Nestle Health Sciences. The other authors have no potential conflicts of interest.

\section{Funding}

None.

\section{Author Contributions}

Conceptualization: all authors; Data curation: ZNM, DJF; Formal analysis: ZNM, DJF; Methodology: KK; Supervision: KK; Validation: KK; Writing-original draft: ZNM, DJF, KK; Writing-review \& editing: all authors.

\section{ORCID}

Zoe N. Memel https://orcid.org/0000-0001-8863-8874 Duncan J. Flynn Yasmin Hernandez-Barco Kavel Harish Visrodia Brenna Casey Kumar Krishnan https://orcid.org/0000-0001-9537-0634 https://orcid.org/0000-0003-1266-3218 https://orcid.org/0000-0002-2762-0889 https://orcid.org/0000-0001-6426-0838 https://orcid.org/0000-0002-9962-8732

\section{REFERENCES}

1. Wendorf KA, Kay M, Baliga C, et al. Endoscopic retrograde cholangiopancreatography-associated AmpC Escherichia coli outbreak. Infect Control Hosp Epidemiol 2015;36:634-642.

2. Kenters N, Huijskens EGW, Meier C, et al. Infectious diseases linked to cross-contamination of flexible endoscopes. Endosc Int Open 


\section{5;3:E259-E265.}

3. Rutala WA, Weber DJ; the Healthcare Infection Control Practices Advisory Committee (HICPAC). Guideline for disinfection and sterilization in healthcare facilities, 2008 [Internet]. Atlanta (GA): Centers for Disease Control and Prevention; 2008 [updated 2019 May 24; cited 2021 Apr 6]. Available from: https://www.cdc.gov/infectioncontrol/guidelines/disinfection/index.html.

4. Alfa MJ, Sepehri S, Olson N, et al. Establishing a clinically relevant bioburden benchmark: a quality indicator for adequate reprocessing and storage of flexible gastrointestinal endoscopes. Am J Infect Control 2012;40:233-236.

5. Bisset L, Cossart YE, Selby W, et al. A prospective study of the efficacy of routine decontamination for gastrointestinal endoscopes and the risk factors for failure. Am J Infect Control 2006;34:274-280.

6. Centers for Disease Control and Prevention (CDC). A rational approach to disinfection and sterilization [Internet]. Atlanta (GA): CDC; 2016 [updated 2016 Sep 18; cited 2021 Apr 6]. Available from: https://www.cdc.gov/infectioncontrol/guidelines/disinfection/rational-approach.html.
7. US Food and Drug Administration (FDA). Reprocessing medical devices in health care settings: validation methods and labeling [Internet]. Silver Spring (MD): FDA; 2015 [updated 2018 Mar 6; cited 2021 Apr 6]. Available from: https://www.fda.gov/regulatory-information/search-fda-guidance-documents/reprocessing-medical-devices-health-care-settings-validation-methods-and-labeling/.

8. Beilenhoff U, Neumann CS, Rey JF, et al. ESGE-ESGENA guideline for quality assurance in reprocessing: microbiological surveillance testing in endoscopy. Endoscopy 2007;39:175-181.

9. Reprocessing Guideline Task Force, Petersen BT, Cohen J, et al. Multisociety guideline on reprocessing flexible GI endoscopes: 2016 update. Gastrointest Endosc 2017;85:282-294.

10. The Association for the Advancement of Medical Instrumentation (AMMI). Standards Spotlight: AAMI TIR99 to provide much-needed guidance for reprocessing dilators and probes [Internet]. Arlington (VA): AMMI; 2018 [updated 2018 Oct 1; cited 2021 Apr 6]. Available from: https://www.aami.org/news/article/StandardsSpotlight-AAMI-TIR99-to-Provide-Much-Needed-Guidance-forReprocessing-Dilators-and-Probes-71335. 